\title{
Alocação de Comprimentos de Ondas em Redes Ópticas usando Otimização por Enxame de Partí-
} culas

\author{
Monteiro, C. C. D \\ Escola Politécnica de Pernambuco \\ Universidade de Pernambuco \\ 50.720-001 - Recife, Brasil \\ Autor1@meu-email
}

\author{
Bastos-Filho, C.J.A \\ Escola Politécnica de Pernambuco \\ Universidade de Pernambuco \\ 50.720-001 - Recife, Brasil \\ Autor2@meu-email
}

Resumo Este artigo propõe o uso de uma técnica de computação inteligente baseada na PSO, que trabalha com permutações em listas para a solução do problema de atribuição de comprimentos de onda em redes ópticas. Os estudos feitos até agora indicam que o uso desta técnica com algumas melhorias podem trazer bons resultados na redução da probabilidade debloqueio na rede..

\footnotetext{
Abstract This paper proposes to use a computation intelligence technique based on PSO, it works with lists permutation to solve the wavelength assignment problem in optical networks. Preliminary results indicate that this technique, with some improvement, could obtain good results in reducing the block probabilities in the network.
} 


\section{Introdução}

Com o objetivo de se estabelecer uma conexão em redes totalmente ópticas [1], um feixe de luz contendo os dados deve percorrer todo o caminho do nó de origem ao de destino. Na maioria das redes atuais, todo esse trajeto tem que ser feito em um mesmo comprimento de onda, devido ao alto custo de tecnologias capazes de converter os comprimentos de onda. Como em um enlace da rede podem passar várias conexões diferentes, em comprimentos de ondas diferentes, é necessário o uso de algum algoritmo que exerça o controle sobre a distribuição dos comprimentos de onda na rede, evitando assim, que uma chamada seja bloqueada.

Durante o processo de estabelecimento de uma chamada, o problema de de roteamento e atribuição de comprimento de onda [3] (do inglês, RWA - routing and wavelength assignment ) onda necessita ser resolvido Este problema costuma ser dividido em duas partes: uma parte para a resolução do roteamento, que trata de encontrar o caminho que a chamada vai percorrer entre os nó de origem e destino; e uma segunda parte referente à atribuição de comprimento de onda, que escolhe por qual canal a chamada vai trafegar.

Neste artigo será proposto o uso da técnica de computação inteligente, conhecida como PSO (Particle Swarm Optimization) [2], para solucionar o problema de atribuição de comprimento (do inglês, Wavelength Assignment). O PSO será usado para determinar a ordem em que os comprimentos serão usados nas chamadas que chegarem na rede.

Ao longo do artigo, serão demonstrados o problema de atribuição de comprimento de onda na seção 2 , uma visão geral sobre o PSO na seção 3, a sua aplicação em redes ópticas na seção 4 e os resultados e conclusão nas seções 5 e 6 .

\section{Problema de Roteamento e Alocação de comprimento de onda.}

Em uma conexão em rede óptica os dados são trafegados por uma fibra óptica através de um feixe de luz, indo de um nó origem a um nó destino. Nas redes ópticas atuais essas fibras apresentam divisão por comprimentos de ondas, permitindo assim que mais de um feixe de luz possa trafegar na fibra ao mesmo tempo.

As redes ópticas podem ser de três tipos, opacas, transparentes e translúcidas. No tipo opaco, em todos os nós da rede é feita uma conversão do sinal óptico para elétrico e de elétrico para óptico novamente para continuar a transmissão, a vantagem dessa abordagem é que em todos os nós é feita uma regeneração no sinal, minimizando assim as perdas nas transmissões, mas em contrapartida gera um congestionamento na rede, diminuindo o seu desempenho, sem aproveitar assim toda a capacidade das fibras ópticas.

Nas redes translúcidas alguns nós apresentam capacidade de tratar o sinal em domínio óptico e outros não, assim sendo o sinal não necessariamente é convertido em todos os nós, atualmente é o tipo de rede mais usada pois apresenta uma desempenho melhor que as opacas e não geram grandes perdas de sinal.

Por fim nas redes transparentes o sinal permanece todo o tempo no domínio óptico, ou seja, todos os nós têm capacidade de tratar sinais ópticos, isso faz com que a rede tenha uma grande capacidade de transmissão de dados, mas em compensação necessite de um grande esforço em estratégias que cuidem da qualidade do sinal, visto que durante todo o caminho ele está exposto a perdas por dispersões, interferências e outros efeitos ópticos.

Nas redes transparentes e translúcidas o sinal óptico pode perder sua qualidade devido as distâncias que ele percorre, e com isso a chamada pode ser perdida. Com isso surge o problema de roteamento e atribuição de comprimento de onda (RWA), que trata de escolher uma rota e um comprimento de onda para a chamada de maneira que diminuam as perdas no sinal, tentando assim minimizar as perdas de dados. O problema RWA costuma ser divididos em dois subproblemas, o de roteamento e o de alocação de comprimento de onda.

\subsection{Roteamento}

No problema de roteamento o interesse é escolher por quais nós a chamada vai passar do nó de origem ao nó de destino (Figura 1), essa escolha tem de maximizar o aproveitamento da rede e minimizar as perdas.

Existem algumas estratégias para a resolução do problema de roteamento, dentre elas estão [6][7]: o roteamento fixo, onde a rota usada para uma chamada entre um dado par de nós é sempre a mesma, um exemplo de roteamento fixo seria o algoritmo de menor caminho, que como seu nome diz escolhe uma rota que possua a menor distância ou custo associado; o roteamento fixo com alternação, onde são usadas uma rota fixa e uma rota que serve como emergência entre dois nós; o roteamento adaptativo, onde a rota entre um par de nós muda dependendo do estado da rede; e o roteamento tolerante a falhas, onde para uma chamada entre dois nós, além da rota fixa pré-determinada existe uma rota de backup, que não tem nenhum laço em comum com a rota anterior, para ser usada em caso de problemas físicos na rota principal. 


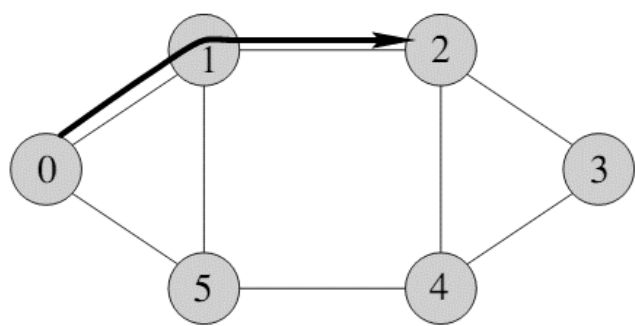

Fig 1. Roteamento de uma chamada entre nós 0 e 2.

\subsection{Atribuição de comprimento de onda (WA)}

No problema de atribuição de comprimento de onda (WA), o foco é decidir por qual comprimento de onda o feixe de luz vai trafegar com os dados, de forma que minimize as penalidades físicas da rede. Em relação ao problema de WA uma informação importante é saber se a rede apresenta capacidade de conversão de comprimento onda em seus nós. Em uma rede que apresente tal capacidade a chamada pode trocar de comprimento de onda de um nó para o outro, continuando o seu caminho normalmente, redes desse tipo facilitam o problema de WA, mas seu uso ainda é muito caro. Em redes que não apresentam essa capacidade a chamada tem de percorrer o seu caminho do nó de origem ao nó de destino em um mesmo comprimento de onda (propriedade da continuidade de comprimento de onda), isto faz com que seja necessário um tratamento especial na questão de WA, para que maximize a quantidade de chamadas que a rede pode aceitar.

No problema de WA existem várias heurísticas, cada uma delas com os seus prós e contras [3][5]. Algumas delas são: random wavelength assignment, first fit, least used, most used, protecting threshold. No random a escolha dos comprimentos de onda é feita de modo randômico, sendo assim um algoritmo de fácil implementação mas de baixo desempenho. O first fit aloca o primeiro comprimento de onda disponível para uma chamada, sendo assim também apresenta resultados similares ao random. O least used aloca as chamadas nos comprimentos de ondas que foram menos usados, tentando balancear a carga entre os comprimentos de onda. No most used ocorre o inverso do anterior, ele tende a concentrar as chamadas nos canais mais usados, deixando assim um maior número de canais livres para uso caso haja necessidade. Por fim o protecting threshold que reserva um limiar de comprimentos de ondas livres, fazendo que para que a chamada seja aceita o número de comprimentos de ondas livres esteja acima desse limiar

\section{Particle Swarm Optimization (PSO)}

Similar a outras abordagens que usam computação inteligente, como Algoritmos Genéticos, o PSO é um método de otimização de funções baseado em populações. $\mathrm{O}$ PSO trabalha basicamente com indivíduos (também chamados de partículas), que compõem a população, e com o fitness de cada indivíduo, um valor numérico que é usado como parâmetro para saber o desempenho de cada indivíduo na função que se deseja fazer a otimização, essa otimização pode ser tanto de maximização quanto de minimização.

Como outras métricas de computação inteligente o PSO é uma abordagem que trabalha com aproximações, ou seja, ela é usada em problemas em que não se sabe uma solução exata. No PSO cada partícula percorre o domínio da função procurando valores que minimizem ou maximizem a função alvo, as partículas mudam a sua posição a cada iteração. Para a troca de posição entre as iterações é usada uma velocidade, que é influenciada pela melhor posição que a partícula já visitou e também pela melhor posição já visitada por todas as partículas, a partir da velocidade a partícula troca a sua posição e então tem o seu fitness avaliado, caso o fitness seja melhor que qualquer já encontrado por essa partícula essa posição passa agora a influenciar na velocidade, e caso o fitness seja melhor do que qualquer fitness já encontrado por qualquer partícula, então todas as partículas terão suas velocidades influenciadas por esta posição.

O PSO funciona com um aprendizado ao longo das iterações, ou seja, para se achar uma otimização de uma função deve-se deixar o PSO rodar uma certa quantidade de iterações, a quantidade de iterações varia entre cada problema, o melhor jeito de determinar é analisando a convergência do algoritmo. Ao fím da sua execução, o PSO terá uma partícula com o melhor fitness da população, esta partícula é a solução aproximada para a função alvo.

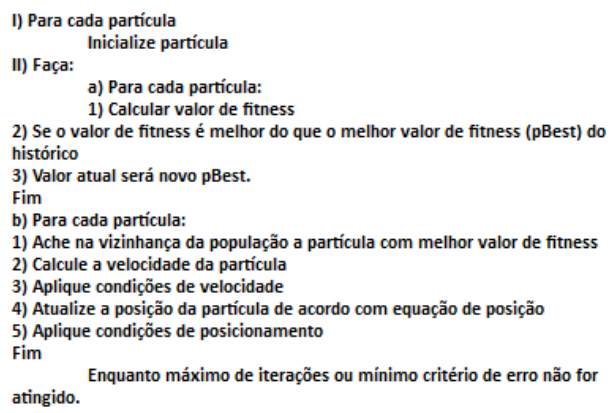

Fig 2. Pseudo-Código do PSO. 


\section{PSO em Redes Ópticas}

O problema de atribuição de redes ópticas também pode ser tratado como um problema de permutações em uma lista de prioridade. Em geral os algoritmos para a solução do problema de WA tem como resultado uma lista que vai conter a ordem em que os comprimentos de onda devem ser usados, ou seja, o número de elementos da lista é o mesmo da quantidade de comprimentos de onda da fibra.

Com isso o uso do PSO para o problema de WA se resume a encontrar uma lista que obtenha o melhor desempenho em uma determinada rede. Algumas mudanças têm de ser feitas no algoritmo tradicional do PSO, enquanto no PSO tradicional a velocidade representa uma distância do espaço de busca que a partícula vai percorrer, no PSO para redes ópticas a velocidade representa uma probabilidade de que ocorra uma permutação naquela posição, caso haja a permutação a partícula permutara seus valores de acordo com a melhor partícula da vizinhança. Para isso, é feita uma normalização no vetor velocidade, e de acordo com as probabilidades vão ocorrendo as permutações em cada posição da lista da partícula.

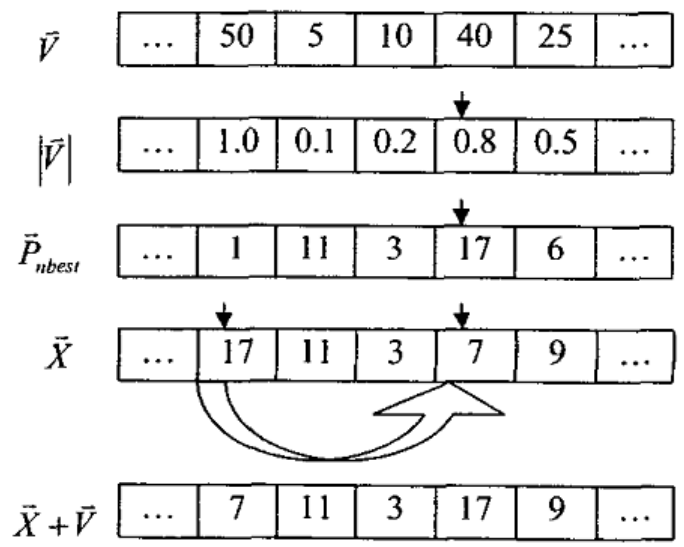

Fig 3. Ilustração do processo de atualização da posição da partícula.

Como as partículas tendem a seguir a melhor na sua vizinhança podem ocorrer de duas partículas se tornarem idênticas e permanecerem eternamente na mesma posição, para tratar desse problema é introduzido um fator de mutação, para caso ocorra de haver duas partículas iguais uma delas escolhe randomicamente duas posições da lista e faz uma permutação entre elas.

O PSO rodará em uma simulação offline para uma dada topologia de rede, e ao fim da simulação terá uma partícula que contém uma lista de ordem para a utilização dos comprimentos de onda, que apresentará os melhores resultados. Para cada rede em que se deseja usar o PSO no problema de WA deve ser feita outra simulação, pois em cada simulação o algoritmo procura por soluções para aquela rede em particular, podendo a solução de uma rede não obter resultados bons em outras.

\section{Metodologia}

Os experimentos realizados para análise dos operadores e parâmetros do algoritmo foram estruturados de maneira que para uma dada topologia da rede e uma configuração do algoritmo a simulação é executado repetidas vezes, para verificar a consistência do algoritmo. As simulações foram feitas na plataforma SIMTON [4], onde o código foi implementado em $\mathrm{C}++$.

As variáveis analisadas no projeto são, a probabilidade de bloqueio nas chamadas da rede e os parâmetros do algoritmo, como o tamanho da população do PSO, tamanho da vizinhança, quantidades de iterações do processo, e outros ajustes possíveis.

Para as simulações foram utilizados dois cenários, ambos na topologia de rede NSFNet e rodando em torno de 10000 cálculos da função objetivo, no primeiro cenário foram utilizadas populações de 12 indivíduos e no segundo cenário populações de 21 indivíduos. Abaixo segue a figura da topologia de rede e dos parâmetros utilizados nas simulações.

\section{Resultados}

As tabelas a seguir mostram os resultados obtidos nos dois cenários estudados, a escolha dessa quantidade de indivíduos para cada simulação se deu a partir do estudo do artigo Swarm intelligence for permutation optimization: a case study of n-queens problem [2], onde é usado uma população com 10 indivíduos, assim o primeiro cenário foi feito com 12 indivíduos e o segundo com 21 para avaliar a possível influência dessa diferença.

Tabela 1. Resultados obtidos nas simulações em cenário com 12 indivíduos.

\begin{tabular}{|l|l|l|}
\hline $\begin{array}{l}\text { No de } \\
\text { Indivíduos }\end{array}$ & $\begin{array}{l}\text { Número de vezes que } \\
\text { a função Objetivo foi } \\
\text { calculada }\end{array}$ & $\begin{array}{l}\text { Probabilidade de } \\
\text { Bloqueio }\end{array}$ \\
\hline 12 & 10236 & 0,145125 \\
\hline 12 & 8364 & 0,127675 \\
\hline 12 & 10488 & 0,1393 \\
\hline 12 & 10452 & 0,15045 \\
\hline 12 & 10488 & 0,1712 \\
\hline
\end{tabular}


A topologia de rede usada foi a NSFNet e os resultados obtidos mostram que o PSO pode vir a ser uma alternativa atrativa para a solução do problema de WA desde que feitas algumas alterações necessárias.

As figras 4 e 5 mostram a curva de convergência da probabilidade de bloqueio da rede em função do número de iterações para 12 e 21 indivíduos, respectivamente.

Tabela 2. Resultados obtidos nas simulações em cenário com 21 indivíduos

\begin{tabular}{|l|l|l|}
\hline $\begin{array}{l}\text { NNo de } \\
\text { Indivíduos }\end{array}$ & $\begin{array}{l}\text { Número de vezes que a } \\
\text { função Objetivo foi } \\
\text { calculada }\end{array}$ & $\begin{array}{l}\text { Probabilidade de } \\
\text { Bloqueio }\end{array}$ \\
\hline 21 & 10458 & 0,145875 \\
\hline 21 & 10458 & 0,151325 \\
\hline 21 & 10437 & 0,131225 \\
\hline 21 & 10479 & 0,1273 \\
\hline
\end{tabular}

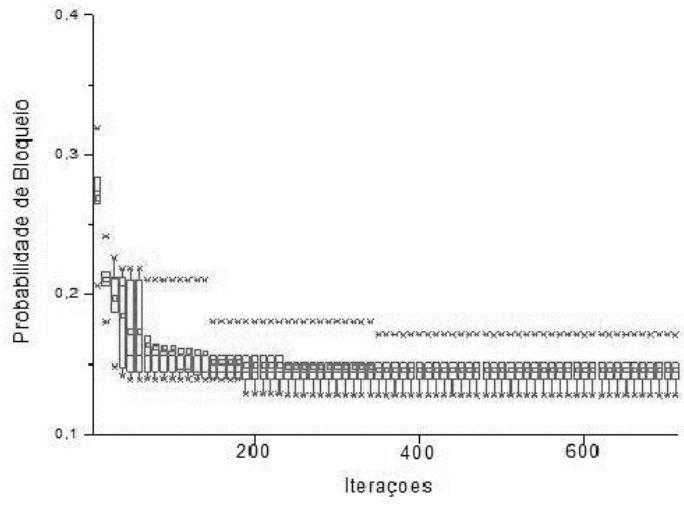

Fig 5. Convergência da Probabilidade de Bloqueio da Rede para 12 indivíduos.

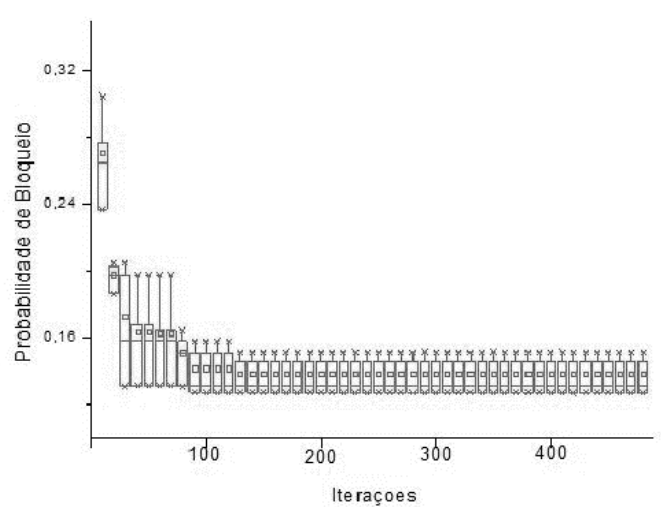

Fig 6. Convergência da Probabilidade de Bloqueio da Rede para 21 indivíduos.

\section{Conclusões}

O propósito deste artigo era demonstrar que o uso de PSO pode ser uma alternativa viável para a resolução do problema de WA, embora no momento atual existam métricas que possuam melhores resultados que o PSO, já fica provado que com um pouco mais de estudos e adaptações o PSO pode alcançar melhores resultados, visto que em outros problemas que tratam de permutações em listas ela já obtêm bons resultados, como no problema das n-Rainhas.

O tamanho da população não pareceu afetar profundamente os resultados obtidos, entretanto sendo necessários maiores estudos para tal confirmação. Devido à rápida convergência do algoritmo os resultados obtidos não foram muito satisfatórios, mas comprovam que o PSO com algumas alterações pode ser usado para resolver problemas de permutação em listas.

\section{Referências}

[1] B. Mukherjee, "WDM Optical Communication Networks:Progress and Challenges," IEEE JSAC, vol. 18 , no. 10 , Oct. 2000 , pp. 1810-24.

[2] H Xiaohui, RC Eberhart, S Yuhui "Swarm Intelligence for Permutation Optimization: A Case Study of n-Queens Problem", IEEE Swarm Intelligence Symposium 2003.

[3] H. Zang, J. Jue, and B. Mukherjee, "A Review of Routing and Wavelength Assignment Approaches for Wavelength Routed Optical WDM Networks", Optical Networks Magazine, January 2000 .

[4] D. A. R. Chaves, C. J. A. Bastos-Filho, J. F. Martins-Filho, "Ferramenta Computacional para Simulação de Redes Ópticas Transparentes". In: MOMAG 2008, 2008, Florianopolis. $13^{\circ} \mathrm{SBMO}$ Simpósio Brasileiro de Microondas e Optoeletrônica e o $8^{\circ}$ CBMag Congresso Brasileiro de Eletromagnetismo - Momag 2008, 2008. v. 1. p. 908913.

[5] D. Bisbal et al, "Dynamic Routing and Wavelength Assignment in Optical Networks by Means of Genetic Algorithms", Photonic Network Communications, 2004, vol. 7, n. 1, pp. 43-58.

[6] D. A. R. Chaves, D. O. Aguiar, C. J. A. BastosFilho, J. F. Martins-Filho, "Novel Adaptive Routing Algorithm for All-Optical Networks Based on 
Power Series and Particle Swarm Optimization". In: Simpósio Brasileiro de Redes de Computadores e Sistemas Distribuídos (SBRC), 2008, Rio de Janeiro. Anais do $26^{\circ}$ Simpósio Brasileiro de Redes de Computadores, 2008.

[7] N. M. Bhide, K. M. Sivalingam, T. Fabry-Asztalos, "Routing mechanisms employing adaptive weight functions for shortest path routing in muilti-wavelenght optical WDM networks", Photon. Netw. Commun. 3, 227-236, 2001. 